\title{
SOME SETS OF DOUBLE LACUNARY INVARIANT SEQUENCES DEFINIED BY FOUR DIMENSIONAL SUMMABLE MATRICES AND ORLICZ FUNCTIONS
}

\author{
AYHAN ESI AND HEMEN DUTTA \\ Received 18 November, 2014
}

\begin{abstract}
The paper introduces some sets of double lacunary invariant sequences defined by four dimensional $R H$-regular matrices and Orlicz functions. First we study some basic properties of such sets by equipping them with linear topological structure. Further, we investigate some relationships among such sets under different conditions and finally compare with a set of double lacunary statistically convergent sequences.
\end{abstract}

2010 Mathematics Subject Classification: 40A05; 40B05

Keywords: Orlicz function, double sequence, $P$-convergence, invariant mean, double lacunary sequence

\section{INTRODUCTION}

An Orlicz function is a function $M:[0, \infty) \rightarrow[0, \infty)$ which is continuous, nondecreasing and convex with $M(0)=0, M(x)>0$ for $x>0$ and $M(x) \rightarrow \infty$ as $x \rightarrow \infty$.

An Orlicz function $M$ is said to satisfy the $\Delta_{2}-$ condition for all values of $u$, if there exists a constant $K>0$, such that $M(2 u) \leq K M(u), u \geq 0$. Note that, if $0<\lambda<1$, then $M(\lambda x) \leq \lambda M(x)$, for all $x \geq 0$.

In 1971 Lindenstrauss and Tzafriri [6] used the idea of Orlicz function to construct the sequence space for single sequences as follows:

$$
l_{M}=\left\{x=\left(x_{k}\right): \sum_{k=1}^{\infty} M\left(\frac{\left|x_{k}\right|}{\rho}\right)<\infty, \text { for some } \rho>0\right\},
$$

which is a Banach space normed by

$$
\left\|\left(x_{k}\right)\right\|=\inf \left\{\rho>0: \sum_{k=1}^{\infty} M\left(\frac{\left|x_{k}\right|}{\rho}\right) \leq 1\right\} .
$$


The Orlicz sequence spaces was further investigated from sequence space point of view and related summability theory by Esi [3], Dutta [2], Tripathy and Dutta [15], Altin et.al. [1] and many others.

Let $\sigma$ be a one-to-one mapping from the set of natural numbers into itself. A continuous linear functional $\phi$ on $\ell_{\infty}$ is said to be an invariant mean or a $\sigma-$ mean provided that

(a) $\phi(x) \geq 0$ when the sequence $x=\left(x_{k}\right)$ is such that $x_{k} \geq 0$ for all $k \in \mathbb{N}$,

(b) $\phi(e)=1$, where $e=(1,1,1, \ldots)$, and

(c) $\phi(x)=\phi\left(x_{\sigma(k)}\right)$ for all $x=\left(x_{k}\right) \in \ell_{\infty}$.

For certain class of mapping $\sigma$ every invariant mean $\varphi$ extends the limit functional on space $c$, the space of all convergent sequences, in sense that $\varphi(x)=\lim x$ for all $x=\left(x_{k}\right) \in c$.

The space $\left[V_{\sigma}\right]$ is of strongly $\sigma$-convergent sequence space was introduced by Mursaleen [8] as follows: A sequence $x=\left(x_{k}\right)$ is said to be strongly $\sigma-$ convergent if there exists a number $\mathrm{L}$ such that

$$
\frac{1}{k} \sum_{i=1}^{k}\left|x_{\sigma^{i}(m)}-L\right| \rightarrow 0 \text { as } k \rightarrow \infty, \text { uniformly in } \mathrm{m} .
$$

If $\sigma(m)=m+1$, then $\left[V_{\sigma}\right]=[\widehat{c}]$, the space of strongly almost convergent sequences, was introduced by Maddox in [7].

A double sequence $x=\left(x_{k, l}\right)$ has a Pringsheim limit $L$ [10] (denoted by $P$ $\lim x=L)$ provided that given an $\varepsilon>0$ there exists an $N \in \mathbb{N}$ such that $\left|x_{k, l}-L\right|<$ $\varepsilon$ whenever $k, l>N$. We shall describe such an $x=\left(x_{k, l}\right)$ more briefly as " $P$ convergent".

The four dimensional matrix $A$ is said to be $R H-$ regular if it maps every bounded $P$-convergent sequence into a $P$-convergent sequence with the same $P$-limit. The assumption of boundedness was made because a double sequence which is $P$-convergent is not necessarily bounded. Using this definition Robison and Hamilton, independently, both presented the following Silverman-Toeplitz type characterization of $\mathrm{RH}$-regularity.

Proposition 1 ([5,11]). The four dimensional matrix $A$ is $R H$-regular if and only if

$$
\begin{aligned}
& R H_{1}: P-\lim _{m, n} a_{m, n, k, l}=0 \text { for each } k \text { and } l ; \\
& R H_{2}: P-\lim _{m, n} \sum_{k, l=1,1}^{\infty, \infty} a_{m, n, k, l}=1 ; \\
& R H_{3}: P-\lim _{m, n} \sum_{k, l=1,1}^{\infty, \infty}\left|a_{m, n, k, l}\right|=0 \text { for each } l ; \\
& R H_{4}: P-\lim _{m, n} \sum_{k, l=1,1}^{\infty, \infty}\left|a_{m, n, k, l}\right|=0, \text { for each } k ; \\
& R H_{5}: \sum_{k, l=1,1}^{\infty}\left|a_{m, n, k, l}\right| \text { is } P-\text { convergent; } \\
& R H_{6}: \text { There exist finite positive integers } A \text { and } B \text { such that } \sum_{k, l>B}\left|a_{m, n, k, l}\right|<
\end{aligned}
$$
$A$. 
The double sequence $\theta_{r, s}=\left\{\left(k_{r}, l_{s}\right)\right\}$ is called double lacunary sequence if there exist two increasing of integers such that

$$
k_{o}=0, h_{r}=k_{r}-k_{r-1} \rightarrow \infty \text { as } r \rightarrow \infty
$$

and

$$
l_{o}=0, \overline{h_{s}}=l_{s}-l_{s-1} \rightarrow \infty \text { as } s \rightarrow \infty .
$$

Notations: $k_{r, s}=k_{r} l_{s}, h_{r, s}=h_{r} \overline{h_{s}}$ and $\theta_{r, s}$ is determined by

$$
\begin{gathered}
I_{r, s}=\left\{(k, l): k_{r-1}<k \leq k_{r} \text { and } l_{s-1}<l \leq l_{s}\right\}, \\
q_{r}=\frac{k_{r}}{k_{r-1}}, \overline{q_{s}}=\frac{l_{s}}{l_{s-1}} \text { and } q_{r, s}=q_{r} \overline{q_{s}} .
\end{gathered}
$$

(see [7]).

The set of all double lacunary sequences denoted by $N_{\theta_{r, s}}$ and defined by Savaş and Patterson [13] as follows:

$$
N_{\theta_{r, s}}=\left\{x=\left(x_{k, l}\right): P-\lim _{r, s} \frac{1}{h_{r, s}} \sum_{(k, l) \in I_{r, s}}\left|x_{k, l}-L\right|=0 \text {, for some L }\right\} \text {. }
$$

For some relevant literature, we also refer to Savaş and Patterson [14].

Let $\theta_{r, s}=\left\{\left(k_{r}, l_{s}\right)\right\}$ be a double lacunary sequence, $M$ be an Orlicz function, $p=\left(p_{k, l}\right)$ be a factorable double sequence of strictly positive real numbers and $A=\left(a_{m, n, k, l}\right)$ be a nonnegative $R H-$ regular summability matrix method. We now define the following new sets of double sequences:

$$
\begin{gathered}
w_{o}^{2}(A, M, p)_{\theta_{r, s}}^{\sigma}= \\
\left\{\begin{array}{c}
x=\left(x_{k, l}\right): P-\lim _{r, s} \frac{1}{h_{r, s}} \sum_{(k, l) \in I_{r, s}} a_{m, n, k, l}\left[M\left(\frac{\left|x_{\sigma^{k}(p), \sigma^{l}(q)}\right|}{\rho}\right)\right]^{p_{k, l}}=0, \\
\text { uniformly in }(p, q), \text { for some } \rho>0
\end{array}\right\}, \\
w^{2}(A, M, p)_{\theta_{r, s}}^{\sigma}= \\
\text { and } \left.\begin{array}{c}
x=\left(x_{k, l}\right): P-\lim _{r, s} \frac{1}{h_{r, s}} \sum_{(k, l) \in I_{r, s}} a_{m, n, k, l}\left[M\left(\frac{\mid x_{\sigma^{k}(p), \sigma^{l}(q)}-L}{\rho}\right)\right]^{p_{k, l}}=0, \\
\text { uniformly in }(p, q), \text { for some } \rho>0 \text { and } L
\end{array}\right\}, \\
w_{\infty}^{2}(A, M, p)_{\theta_{r, s}}^{\sigma}= \\
\left\{\begin{array}{c}
x=\left(x_{k, l}\right): \sup _{m, n, r, s, p, q} \frac{1}{h_{r, s}} \sum_{(k, l) \in I_{r, s}} a_{m, n, k, l}\left[M\left(\frac{\mid x_{\sigma^{k}(p), \sigma l} l(q)}{\rho}\right)\right]^{p_{k, l}}<\infty, \\
\text { for some } \rho>0
\end{array}\right\} .
\end{gathered}
$$

and

When $M(x)=x$, for all $x \in[0, \infty)$ we have the following sets of double sequences:

$$
w_{o}^{2}(A, p)_{\theta_{r, s}}^{\sigma}=
$$




$$
\left.\begin{array}{c}
\left\{x=\left(x_{k, l}\right): P-\lim _{r, s} \frac{1}{h_{r, s}} \sum_{(k, l) \in I_{r, s}} a_{m, n, k, l}\left|x_{\sigma^{k}(p), \sigma^{l}(q)}\right|^{p_{k, l}}=0,\right. \\
\text { uniformly in }(p, q)
\end{array}\right\},
$$

and

$$
\begin{gathered}
w_{\infty}^{2}(A, p)_{\theta_{r, s}}^{\sigma}= \\
\left\{x=\left(x_{k, l}\right): \sup _{m, n, r, s, p, q} \frac{1}{h_{r, s}} \sum_{(k, l) \in I_{r, s}} a_{m, n, k, l}\left|x_{\sigma^{k}(p), \sigma^{l}(q)}\right|^{p_{k, l}}<\infty\right\} .
\end{gathered}
$$

When $\sigma(p)=p+1$ and $\sigma(q)=q+1$, we have the following sets of double sequences:

$$
\begin{gathered}
\widehat{w}_{o}^{2}(A, M, p)_{\theta_{r, s}}= \\
\left\{\begin{array}{c}
x=\left(x_{k, l}\right): P-\lim _{r, s} \frac{1}{h_{r, s}} \sum_{(k, l) \in I_{r, s}} a_{m, n, k, l}\left[M\left(\frac{\left|x_{k+p, l+q}\right|}{\rho}\right)\right]^{p_{k, l}}=0, \\
\text { uniformly in }(p, q), \text { for some } \rho>0
\end{array}\right\}, \\
\widehat{w}^{2}(A, M, p)_{\theta_{r, s}}= \\
\left\{\begin{array}{c}
x=\left(x_{k, l}\right): P-\lim _{r, s} \frac{1}{h_{r, s}} \sum_{(k, l) \in I_{r, s}} a_{m, n, k, l}\left[M\left(\frac{\left|x_{k+p, l+q}-L\right|}{\rho}\right)\right]^{p_{k, l}}=0, \\
\text { uniformly in }(p, q), \text { for some } \rho>0 \text { and } L
\end{array}\right\},
\end{gathered}
$$

and

$$
\left.\begin{array}{c}
\widehat{w}_{\infty}^{2}(A, M, p)_{\theta_{r, s}}= \\
\left\{x=\left(x_{k, l}\right): \sup _{m, n, r, s, p, q} \frac{1}{h_{r, s}} \sum_{(k, l) \in I_{r, s}} a_{m, n, k, l}\left[M\left(\frac{\left|x_{k+p, l+q}\right|}{\rho}\right)\right]^{p_{k, l}}<\infty,\right. \\
\text { for some } \rho>0
\end{array}\right\} .
$$

When $p_{k, l}=1$, for all $k, l \in \mathbb{N}$, we obtain the following sets of double sequences:

$$
\begin{aligned}
& w_{o}^{2}(A, M)_{\theta_{r, s}}^{\sigma}=
\end{aligned}
$$

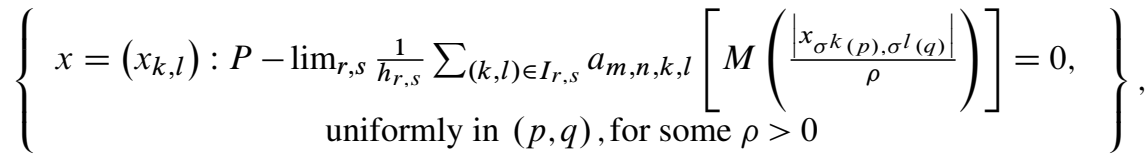

$$
\begin{aligned}
& w^{2}(A, M)_{\theta_{r, s}}^{\sigma}= \\
& \left\{\begin{array}{c}
x=\left(x_{k, l}\right): P-\lim _{r, s} \frac{1}{h_{r, s}} \sum_{(k, l) \in I_{r, s}} a_{m, n, k, l}\left[M\left(\frac{\left|x_{\sigma^{k}(p), \sigma^{l}(q)}-L\right|}{\rho}\right)\right]=0, \\
\text { uniformly in }(p, q), \text { for some } \rho>0 \text { and } L
\end{array}\right\},
\end{aligned}
$$

and

$$
w_{\infty}^{2}(A, M)_{\theta_{r, s}}^{\sigma}=
$$




$$
\left\{\begin{array}{c}
x=\left(x_{k, l}\right): \sup _{m, n, r, s, p, q} \frac{1}{h_{r, s}} \sum_{(k, l) \in I_{r, s}} a_{m, n, k, l}\left[M\left(\frac{\left|x_{\sigma^{k}(p), \sigma^{l}(q)}\right|}{\rho}\right)\right]<\infty, \\
\text { for some } \rho>0
\end{array}\right\} .
$$

\section{MAIN RESULTS}

In this section we shall investigate relevant properties of the sets defined in previous sections.

Theorem 1. Let $p=\left(p_{k, l}\right)$ be bounded. The classes of sequences $w_{o}^{2}(A, M, p)_{\theta_{r, s}}^{\sigma}$, $w^{2}(A, M, p)_{\theta_{r, s}}^{\sigma}$ and $w_{\infty}^{2}(A, M, p)_{\theta_{r, s}}^{\sigma}$ are linear spaces.

Proof. It is easy, so we omit it.

Theorem 2. Let $0<h=\inf p_{k, l} \leq \sup p_{k, l}=H<\infty$, and let $A$ is a nonnegative $R H$ - regular summability matrix method. For any Orlicz function $M$, if $\beta=$ $\lim _{t \rightarrow \infty} \frac{M(t)}{t} \geq 1$, then $w^{2}(A, p)_{\theta_{r, s}}^{\sigma}=w^{2}(A, M, p)_{\theta_{r, s}}^{\sigma}$.

Proof. Let $0<h=\inf p_{k, l} \leq \sup p_{k, l}=H<\infty$ and $x=\left(x_{k, l}\right) \in w^{2}(A, p)_{\theta_{r, s}}^{\sigma}$ and let $0<\varepsilon<1$ and $\delta$ with $0<\delta<1$ such that $M(t)<\varepsilon$ for $0 \leq t<\delta$. We can write for each $m$ and $n$

$$
\begin{aligned}
& \sum_{(k, l) \in I_{r, s}} a_{m, n, k, l}\left[M\left(\frac{\left|x_{\sigma^{k}(p), \sigma^{l}(q)}-L\right|}{\rho}\right)\right]^{p_{k, l}} \\
= & \sum_{\substack{(k, l) \in I_{r, s} \\
x_{\sigma^{k}(p), \sigma^{l}(q)}-L \mid \leq \delta}} a_{m, n, k, l}\left[M\left(\frac{\left|x_{\sigma^{k}(p), \sigma^{l}(q)}-L\right|}{\rho}\right)\right]^{p_{k, l}} \\
+ & \sum_{\substack{(k, l) \in I_{r, s}\\
}} a_{m, n, k, l}\left[M\left(\frac{\left|x_{\sigma^{k}(p), \sigma^{l}(q)}-L\right|}{\rho}\right)\right]^{p_{k, l}} .
\end{aligned}
$$

Then

$$
\begin{gathered}
\sum_{\substack{(k, l) \in I_{r, s} \\
\left|x_{\sigma^{k}(p), \sigma^{l}(q)}-L\right| \leq \delta}} a_{m, n, k, l}\left[M\left(\frac{\left|x_{\sigma^{k}(p), \sigma^{l}(q)}-L\right|}{\rho}\right)\right]^{p_{k, l}} \\
\leq \varepsilon^{h} \sum_{(k, l) \in I_{r, s}} a_{m, n, k, l}
\end{gathered}
$$


On the other hand, we use the fact that

$$
\left|x_{\sigma^{k}(p), \sigma^{l}(q)}-L\right|<1+\left[\left|\frac{\left|x_{\sigma^{k}(p), \sigma^{l}(q)}-L\right|}{\rho}\right|\right]
$$

where $[|t|]$ denotes the integer part of $t$. Since $M$ is Orlicz function we have

$$
M\left(\frac{\left|x_{\sigma^{k}(p), \sigma^{l}(q)}-L\right|}{\rho}\right) \geq M(1) .
$$

Now, let us consider the second part where the sum is taken over $\left|x_{\sigma^{k}(p), \sigma^{l}(q)}-L\right|>$ $\delta$.

Thus

$$
\begin{aligned}
& \quad \sum_{\substack{(k, l) \in I_{r, s} \\
\left|x_{\sigma^{k}(p), \sigma^{l}(q)}-L\right|>\delta}} a_{m, n, k, l}\left[M\left(\frac{\left|x_{\sigma^{k}(p), \sigma^{l}(q)}-L\right|}{\rho}\right)\right]^{p_{k, l}} \\
& \leq \sum_{\substack{(k, l) \in I_{r, s} \\
\left|x_{\sigma^{k}(p), \sigma^{l}(q)}-L\right|>\delta}} a_{m, n, k, l}\left[M\left(1+\left[\left|\frac{\left|x_{\sigma^{k}(p), \sigma^{l}(q)}-L\right|}{\rho}\right|\right]\right)\right]^{p_{k, l}} \\
& \leq\left(2 M(1) \delta^{-1}\right)^{H} \sum_{(k, l) \in I_{r, s}} a_{m, n, k, l}\left(\frac{\left|x_{\sigma^{k}(p), \sigma^{l}(q)}-L\right|}{\rho}\right)^{p_{k, l}}
\end{aligned}
$$

This and from (2.1) and $R H-$ regularity of $A$, we are granted that $x=\left(x_{k, l}\right) \in$ $w^{2}(A, M, p)_{\theta_{r, s}}^{\sigma}$. Observe that in this part of the proof we did not use $\beta \geq 1$. Let $\beta \geq 1$ and $x=\left(x_{k, l}\right) \in w^{2}(A, M, p)_{\theta_{r, s}}^{\sigma}$. Since $\beta \geq 1$ we have $M(t) \geq \beta t$ for all $t \geq 0$. It follows that $x=\left(x_{k, l}\right) \in w^{2}(A, M, p)_{\theta_{r, s}}^{\sigma}$ implies $x=\left(x_{k, l}\right) \in w^{2}(A, p)_{\theta_{r, s}}^{\sigma}$ and this completes the proof.

Theorem 3. $w_{o}^{2}(A, M, p)_{\theta_{r, s}}^{\sigma}, w^{2}(A, M, p)_{\theta_{r, s}}^{\sigma}$ and $w_{\infty}^{2}(A, M, p)_{\theta_{r, s}}^{\sigma}$ are complete linear topological spaces with the paranorm

$$
\begin{gathered}
g\left(\left(x_{k, l}\right)\right)= \\
\inf \left\{\rho^{\frac{p_{k, l}}{T}}>0: \sup _{m, n, r, s, p, q}\left(\frac{1}{h_{r, s}} \sum_{(k, l) \in I_{r, s}} a_{m, n, k, l}\left[M\left(\frac{\left|x_{\sigma^{k}(p), \sigma^{l}(q)}\right|}{\rho}\right)\right]^{p_{k, l}}\right)^{\frac{1}{T}} \leq 1\right\}
\end{gathered}
$$

where $T=\max (1, H), H=\sup _{k, l} p_{k, l}<\infty$.

Proof. Clearly $g(0)=0, g(-x)=g(x)$. 
Let $x=\left(x_{k, l}\right), y=\left(y_{k, l}\right) \in w_{\infty}^{2}(A, M, p)_{\theta_{r, s}}^{\sigma}$. Then there exist some $\rho_{1}$ and $\rho_{2}$ such that

$$
\sup _{m, n, r, s, p, q}\left(\frac{1}{h_{r, s}} \sum_{(k, l) \in I_{r, s}} a_{m, n, k, l}\left[M\left(\frac{\left|x_{\sigma^{k}(p), \sigma^{l}(q)}\right|}{\rho}\right)\right]^{p_{k, l}}\right)^{\frac{1}{T}}
$$

and

$$
\sup _{m, n, r, s, p, q}\left(\frac{1}{h_{r, s}} \sum_{(k, l) \in I_{r, s}} a_{m, n, k, l}\left[M\left(\frac{\mid y_{\sigma^{k}}(p), \sigma^{l}(q)}{\rho}\right)\right]^{p_{k, l}}\right)^{\frac{1}{T}} .
$$

Let $\rho=\rho_{1}+\rho_{2}$. Then we have

$$
\begin{gathered}
\sup _{m, n, r, s, p, q}\left(\frac{1}{h_{r, s}} \sum_{(k, l) \in I_{r, s}} a_{m, n, k, l}\left[M\left(\frac{\left|x_{\sigma^{k}(p), \sigma^{l}(q)}+y_{\sigma^{k}(p), \sigma^{l}(q)}\right|}{\rho}\right)\right]^{p_{k, l}}\right)^{\frac{1}{T}} \\
\sup _{m, n, r, s, p, q}\left(\frac{1}{h_{r, s}} \sum_{(k, l) \in I_{r, s}} a_{m, n, k, l}\left[M\left(\frac{\left|x_{\sigma^{k}(p), \sigma^{l}(q)}+y_{\sigma^{k}(p), \sigma^{l}(q)}\right|}{\rho_{1}+\rho_{2}}\right)\right]^{p_{k, l}}\right)^{\frac{1}{T}} \\
\leq \sup _{m, n, r, s, p, q}\left(\frac { 1 } { h _ { r , s } } \sum _ { ( k , l ) \in I _ { r , s } } a _ { m , n , k , l } \left[\frac{\rho_{1}}{\rho_{1}+\rho_{2}} M\left(\frac{\left|x_{\sigma^{k}(p), \sigma^{l}(q)}\right|}{\rho_{1}}\right)\right.\right. \\
\left.+\frac{\rho_{2}}{\rho_{1}+\rho_{2}} M\left(\frac{\left|y_{\sigma^{k}(p), \sigma^{l}(q)}\right|}{\rho_{2}}\right)\right]^{\left.p_{k, l}\right)^{\frac{1}{T}}}
\end{gathered}
$$

By Minkowsky's inequality

$$
\begin{aligned}
& \leq\left(\frac{\rho_{1}}{\rho_{1}+\rho_{2}}\right) \sup _{m, n, r, s, p, q}\left(\frac{1}{h_{r, s}} \sum_{(k, l) \in I_{r, s}} a_{m, n, k, l}\left[M\left(\frac{\left|x_{\sigma^{k}(p), \sigma^{l}(q)}\right|}{\rho_{1}}\right)\right]^{p_{k, l}}\right)^{\frac{1}{T}} \\
& +\left(\frac{\rho_{2}}{\rho_{1}+\rho_{2}}\right) \sup _{m, n, r, s, p, q}\left(\frac{1}{h_{r, s}} \sum_{(k, l) \in I_{r, s}} a_{m, n, k, l}\left[M\left(\frac{\left|y_{\sigma^{k}(p), \sigma^{l}(q)}\right|}{\rho_{2}}\right)\right]^{p_{k, l}}\right)^{\frac{1}{T}} \leq 1 .
\end{aligned}
$$

Now

$$
g\left(\left(x_{k, l}\right)+\left(y_{k, l}\right)\right)=\inf \left\{\rho^{\frac{p_{k, l}}{T}}>0:\right.
$$

$$
\begin{gathered}
\left.\sup _{m, n, r, s, p, q}\left(\frac{1}{h_{r, s}} \sum_{(k, l) \in I_{r, s}} a_{m, n, k, l}\left[M\left(\frac{\left|x_{\sigma^{k}(p), \sigma^{l}(q)}+y_{\sigma^{k}(p), \sigma^{l}(q)}\right|}{\rho}\right)\right]^{p_{k, l}}\right)^{\frac{1}{T}} \leq 1\right\} \\
\leq \\
\inf \left\{\rho_{1}^{\frac{p_{k, l}}{T}}>0: \sup _{m, n, r, s, p, q}\left(\frac{1}{h_{r, s}} \sum_{(k, l) \in I_{r, s}} a_{m, n, k, l}\left[M\left(\frac{\left|x_{\sigma^{k}(p), \sigma^{l}(q)}\right|}{\rho_{1}}\right)\right]^{p_{k, l}}\right)^{\frac{1}{T}} \leq 1\right\}
\end{gathered}
$$




$$
\begin{gathered}
\inf \left\{\rho_{2}^{\frac{p_{k, l}}{T}}>0: \sup _{m, n, r, s, p, q}\left(\frac{1}{h_{r, s}} \sum_{(k, l) \in I_{r, s}} a_{m, n, k, l}\left[M\left(\frac{\left|y_{\sigma^{k}(p), \sigma^{l}(q)}\right|}{\rho_{2}}\right)\right]^{p_{k, l}}\right)^{\frac{1}{T}} \leq 1\right\} \\
=g\left(\left(x_{k, l}\right)\right)+g\left(\left(y_{k, l}\right)\right) .
\end{gathered}
$$

Let $\lambda \in \mathbb{C}$, then the continuity of the product follows from the following equality:

$$
\begin{gathered}
g\left(\lambda\left(x_{k, l}\right)\right)=\inf \left\{\rho^{\frac{p_{k, l}}{T}}>0:\right. \\
\left.\sup _{m, n, r, s, p, q}\left(\frac{1}{h_{r, s}} \sum_{(k, l) \in I_{r, s}} a_{m, n, k, l}\left[M\left(\frac{\left|\lambda x_{\sigma^{k}(p), \sigma^{l}(q)}\right|}{\rho}\right)\right]^{p_{k, l}}\right)^{\frac{1}{T}} \leq 1, \rho>0\right\} \\
=\inf \left\{(|\lambda| r)^{\frac{p_{k, l}}{T}}>0:\right. \\
\left.\sup _{m, n, r, s, p, q}\left(\frac{1}{h_{r, s}} \sum_{(k, l) \in I_{r, s}} a_{m, n, k, l}\left[M\left(\frac{\left|\lambda x_{\sigma^{k}(p), \sigma^{l}(q)}\right|}{\rho}\right)\right]^{p_{k, l}}\right)^{\frac{1}{T}} \leq 1, r>0\right\} \\
=|\lambda| g\left(\left(x_{k, l}\right)\right),
\end{gathered}
$$

where $\frac{1}{r}=\frac{|\lambda|}{\rho}$.

Now $\left(x_{k, l}^{s}\right)$ is a Cauchy sequence in $w_{\infty}^{2}(A, M, p)_{\theta_{r, s}}^{\sigma}$. Then

$$
g\left(\left(x_{k, l}^{s}-x_{k, l}^{t}\right)\right) \rightarrow 0 \text { as } s, t \rightarrow \infty .
$$

For given $\varepsilon>0$, choose $r>0$ and $x_{o}>0$ be such that $\frac{\varepsilon}{r x_{o}}>0$ and $M\left(\frac{r x_{o}}{2}\right) \geq 1$. Now $g\left(\left(x_{k, l}^{s}-x_{k, l}^{t}\right)\right) \rightarrow 0$ as $s, t \rightarrow \infty$ implies that there exists $n_{o} \in \mathbb{N}$ such that

$$
g\left(\left(x_{k, l}^{s}-x_{k, l}^{t}\right)\right)<\frac{\varepsilon}{r x_{o}} \text { for all } s, t \geq n_{o} .
$$

This implies

$$
\begin{aligned}
\inf \left\{\rho^{\frac{p_{k, l}}{T}}>0:\right. & \left.\sup _{m, n, r, s, p, q}\left(\frac{1}{h_{r, s}} \sum_{(k, l) \in I_{r, s}} a_{m, n, k, l}\left[M\left(\frac{\left|x_{\sigma^{k}(p), \sigma^{l}(q)}^{s}-x_{\sigma^{k}(p), \sigma^{l}(q)}^{t}\right|}{\rho}\right)\right]^{p_{k, l}}\right)^{\frac{1}{T}} \leq 1\right\} \\
& <\frac{\varepsilon}{r x_{o}}
\end{aligned}
$$


Now from (2.2) we have,

$$
\begin{aligned}
& M\left(\frac{\left|x_{\sigma^{k}(p), \sigma^{l}(q)}^{s}-x_{\sigma^{k}(p), \sigma^{l}(q)}^{t}\right|}{\rho}\right) \leq 1 \leq M\left(\frac{r x_{o}}{2}\right) \\
& \Rightarrow \frac{\left|x_{\sigma^{k}(p), \sigma^{l}(q)}^{s}-x_{\sigma^{k}(p), \sigma^{l}(q)}^{t}\right|}{g\left(\left(x_{k, l}^{s}-x_{k, l}^{t}\right)\right)} \leq \frac{r x_{o}}{2} \\
& \Rightarrow\left|x_{\sigma^{k}(p), \sigma^{l}(q)}^{s}-x_{\sigma^{k}(p), \sigma^{l}(q)}^{t}\right|<\frac{r x_{o}}{2} \cdot \frac{\varepsilon}{r x_{o}}=\frac{\varepsilon}{2} .
\end{aligned}
$$

This implies that $\left(x_{\sigma^{k}(p), \sigma^{l}(q)}^{s}\right)$ is a Cauchy sequence of real numbers.

Let $\lim _{s \rightarrow \infty} x_{\sigma^{k}(p), \sigma^{l}(q)}^{s}=x_{\sigma^{k}(p), \sigma^{l}(q)}$ for all $k, l \in N$. Using continuity of $M$, we have

$$
\begin{aligned}
& \lim _{t \rightarrow \infty} M\left(\frac{\left|x_{\sigma^{k}(p), \sigma^{l}(q)}^{s}-x_{\sigma^{k}(p), \sigma^{l}(q)}^{t}\right|}{\rho}\right) \leq 1 \\
& \Rightarrow M\left(\frac{\left|x_{\sigma^{k}(p), \sigma^{l}(q)}^{s}-x_{\sigma^{k}(p), \sigma^{l}(q)}\right|}{\rho}\right) \leq 1 .
\end{aligned}
$$

Let $s \geq n_{o}$, then taking infimum of such $\rho^{\prime} s$ we have $g\left(\left(x_{k, l}^{s}-x_{k, l}\right)\right)<\varepsilon$. Thus $\left(x_{k, l}^{s}-x_{k, l}\right) \in w_{\infty}^{2}(A, M, p)_{\theta_{r, s}}^{\sigma}$. By linearity of the space $w_{\infty}^{2}(A, M, p)_{\theta_{r, s}}^{\sigma}$ we have $\left(x_{k, l}\right) \in w_{\infty}^{2}(A, M, p)_{\theta_{r, s}}^{\sigma}$. Hence $w_{\infty}^{2}(A, M, p)_{\theta_{r, s}}^{\sigma}$ is a complete space.

Proposition 2. We have the following inclusions

(a) $w^{2}(A, M, p)_{\theta_{r, s}}^{\sigma} \subset w_{\infty}^{2}(A, M, p)_{\theta_{r, s}}^{\sigma}$,

(b) $w_{o}^{2}(A, M, p)_{\theta_{r, s}}^{\sigma,} \subset w_{\infty}^{2}(A, M, p)_{\theta_{r, s}}^{\sigma, s}$.

Proof. It is easy, so we omit it.

Theorem 4. The spaces $w_{o}^{2}(A, M, p)_{\theta_{r, s}}^{\sigma}$ and $w^{2}(A, M, p)_{\theta_{r, s}}^{\sigma}$ are nowhere dense subsets of $w_{\infty}^{2}(A, M, p)_{\theta_{r, s}}^{\sigma}$.

Proof. The proof is clear in view of Theorem 3 and Proposition 2.

Theorem 5. (a) If $0<h=\inf p_{k, l}<p_{k, l} \leq 1$, then

$$
w^{2}(A, M, p)_{\theta_{r, s}}^{\sigma} \subset w^{2}(A, M)_{\theta_{r, s}}^{\sigma} .
$$

(b) If $1 \leq p_{k, l} \leq \sup p_{k, l}<\infty$, then

$$
w^{2}(A, M)_{\theta_{r, s}}^{\sigma} \subset w^{2}(A, M, p)_{\theta_{r, s}}^{\sigma} .
$$


Proof. (a) Let $x=\left(x_{k, l}\right) \in w^{2}(A, M, p)_{\theta_{r, S}}$, since $0<h=\inf p_{k, l}<p_{k, l} \leq 1$, we obtain the following:

$$
\begin{aligned}
& \sum_{(k, l) \in I_{r, s}} a_{m, n, k, l} M\left(\frac{\left|x_{\sigma^{k}(p), \sigma^{l}(q)}-L\right|}{\rho}\right) \\
\leq & \sum_{(k, l) \in I_{r, s}} a_{m, n, k, l}\left[M\left(\frac{\left|x_{\sigma^{k}(p), \sigma^{l}(q)}-L\right|}{\rho}\right)\right]^{p_{k, l}}
\end{aligned}
$$

thus $x=\left(x_{k, l}\right) \in w^{2}(A, M)_{\theta_{r, s}}^{\sigma}$.

(b) Let $p_{k, l} \geq 1$ for each $k, l$ and $\sup p_{k, l}<\infty$ and let $x=\left(x_{k, l}\right) \in w^{2}(A, M)_{\theta_{r, s}}^{\sigma}$. Then for each $0<\varepsilon<1$ there exists a positive integer $K$ such that

$$
\sum_{(k, l) \in I_{r, s}} a_{m, n, k, l} M\left(\frac{\left|x_{\sigma^{k}(p), \sigma^{l}(q)}-L\right|}{\rho}\right) \leq \varepsilon<1
$$

for all $n, m \geq K$. This implies that

$$
\begin{aligned}
& \sum_{(k, l) \in I_{r, s}} a_{m, n, k, l}\left[M\left(\frac{\left|x_{\sigma^{k}(p), \sigma^{l}(q)}-L\right|}{\rho}\right)\right]^{p_{k, l}} \\
\leq & \sum_{(k, l) \in I_{r, s}} a_{m, n, k, l} M\left(\frac{\left|x_{\sigma^{k}(p), \sigma^{l}(q)}-L\right|}{\rho}\right) .
\end{aligned}
$$

Thus $x=\left(x_{k, l}\right) \in w^{2}(A, M, p)_{\theta_{r, s}}^{\sigma}$. This completes the proof.

\section{Double STATISTICAl CONVERGENCE}

The concept of statistical convergence for single sequences was introduced by Fast [4] in 1951. Later, Mursaleen and Edely [9] defined the statistical analogue for double sequence $x=\left(x_{k, l}\right)$ as follows: A real double sequence $x=\left(x_{k, l}\right)$ is said to be $P$-statistical convergence to $L$ provided that for each $\varepsilon>0$

$$
P-\lim _{m, n} \frac{1}{m n}\left|\left\{(k, l) \in \mathbb{N} \times \mathbb{N}: k \leq m, l \leq n ;\left|x_{k, l}-L\right| \geq \varepsilon\right\}\right|=0
$$

where the vertical bars indicate the numbers of elements in the enclosed set. In this case, we write $s t_{2}-\lim _{k, l} x_{k, l}=L$ and we denote the set of all $P$-statistical convergent double sequences by $s_{2}$.

Definition 1 ([12]). Let $\theta_{r, s}=\left\{\left(k_{r}, l_{s}\right)\right\}$ be a double lacunary sequence, a real double sequence $x=\left(x_{k, l}\right)$ is said to be uniformly $S_{(\theta, \sigma)}^{2}-$ convergent or uniformly $(\theta, \sigma)$-convergence to $L$ provided that for each $\varepsilon>0$

$$
P-\lim _{r, s} \frac{1}{h_{r, s}} \max _{p, q}\left|\left\{(k, l) \in I_{r, s}:\left|x_{\sigma^{k}(p), \sigma^{l}(q)}-L\right| \geq \varepsilon\right\}\right|=0 .
$$


In this case, we write $S_{(\theta, \sigma)}^{2}-\lim _{k, l} x_{k, l}=L$ and we denote the set of all $P-$ statistical $S_{(\theta, \sigma)}^{2}$-convergent double sequences by $S_{(\theta, \sigma)}^{2}$.

In this section we give some relationship between the double sequence spaces $S_{(\theta, \sigma)}^{2}$ and $w^{2}(M)_{\theta_{r, s}}^{\sigma}$

Theorem 6. If $M$ be an Orlicz function, then $w^{2}(M)_{\theta_{r, s}}^{\sigma} \subset S_{(\theta, \sigma)}^{2}$, where

$$
w^{2}(M)_{\theta_{r, s}}^{\sigma}=\left\{\begin{array}{c}
x=\left(x_{k, l}\right): P-\lim _{r, s} \frac{1}{h_{r, s}} \sum_{(k, l) \in I_{r, s}} M\left(\frac{\left|x_{\sigma^{k}(p), \sigma^{l}(q)}-L\right|}{\rho}\right)=0, \\
\text { uniformly in }(p, q), \text { for some } \rho>0 \text { and } L
\end{array}\right\}
$$

Proof. Suppose that $x=\left(x_{k, l}\right) \in w^{2}(M)_{\theta_{r, s}}^{\sigma}$ and $\varepsilon>0$, then we obtain the following for every $p$ and $q$

$$
\begin{gathered}
\frac{1}{h_{r, s}} \sum_{(k, l) \in I_{r, s}} M\left(\frac{\left|x_{\sigma^{k}(p), \sigma^{l}(q)}-L\right|}{\rho}\right) \geq \frac{1}{h_{r, s}} \sum_{\substack{(k, l) \in I_{r, s} \\
\left|x_{\sigma^{k}(p), \sigma^{l}(q)}-L\right| \geq \varepsilon}} M\left(\frac{\left|x_{\sigma^{k}(p), \sigma^{l}(q)}-L\right|}{\rho}\right) \\
\geq \frac{M(\varepsilon)}{h_{r, s}}\left|\left\{(k, l) \in I_{r, s}:\left|x_{\sigma^{k}(p), \sigma^{l}(q)}-L\right| \geq \varepsilon\right\}\right| .
\end{gathered}
$$

Hence $x=\left(x_{k, l}\right) \in S_{(\theta, \sigma)}^{2}$.

Theorem 7. Let $M$ be an Orlicz function. Then $S_{(\theta, \sigma)}^{2} \cap \ell_{\infty}^{2} \subset w^{2}(M)_{\theta_{r, s}}^{\sigma}$.

Proof. Let $x=\left(x_{k, l}\right) \in S_{(\theta, \sigma)}^{2} \cap \ell_{\infty}^{2}$. Since $x \in \ell_{\infty}^{2}$, we can find a positive number $K$ such that $M(x) \leq K$, for all $x \geq 0$. Then for each $p$ and $q$, we have

$$
\begin{aligned}
& \frac{1}{h_{r, s}} \sum_{(k, l) \in I_{r, s}} M\left(\frac{\mid x_{\sigma^{k}}(p), \sigma^{l}(q)}{\rho}\right) L \mid \\
& =\frac{1}{h_{r, s}} \sum_{\substack{(k, l) \in I_{r, s} \\
\left|x_{\sigma^{k}(p), \sigma^{l}(q)}-L\right| \geq \varepsilon}} M\left(\frac{\left|x_{\sigma^{k}(p), \sigma^{l}(q)}-L\right|}{\rho}\right) \\
& +\frac{1}{h_{r, s}} \sum_{(k, l) \in I_{r, s}} M\left(\frac{\left|x_{\sigma^{k}(p), \sigma^{l}(q)}-L\right|}{\rho}\right) \\
& \leq \frac{K}{h_{r, s}}\left|\left\{(k, l) \in I_{r, s}:\left|x_{\sigma^{k}}(p), \sigma^{l}(q)-L\right| \geq \varepsilon\right\}\right|+M(\varepsilon)
\end{aligned}
$$

and thus the Pringsheim's limit on $r$ and $s$ grant us the result. 


\section{REFERENCES}

[1] Y. Altin, M. Et, and B. Tripathy, "On sequence space $\left|\bar{N}_{p}\right|(m, r, q, s)$ on seminormed spaces," App. Math. Comput., vol. 154, pp. 423-430, 2004, doi: 10.1016/S0096-3003(03)00722-7.

[2] H. Dutta, "A characterization of the class of statistically pre-Cauchy double sequences of fuzzy numbers," App. Math. Inf. Sci., vol. 7, no. 4, pp. 1437-1440, 2013, doi: 10.12785/amis/070423.

[3] A. Esi, "Some new sequence spaces defined by Orlicz functions," Bull. Inst. Math., Academia Sinica, vol. 27, no. 1, pp. 71-76, 1999.

[4] H. Fast, "Sur la convergence statistique," Colloq. Math., vol. 2, pp. 241-244, 1951.

[5] H. Hamilton, "Transformations of multiple sequences," Duke Math. J., vol. 2, pp. 29-60, 1936, doi: 10.1215/S0012-7094-36-00204-1.

[6] J. Lindenstrauss and L. Tzafriri, “On Orlicz sequence spaces,” Israel J. Math., vol. 10, pp. 379390, 1971, doi: 10.1007/BF02771656.

[7] I. Maddox, "Spaces of strongly summable sequences," Quart. J. Math., Oxford Ser., vol. 18, no. 2, pp. 345-355, 1967, doi: 10.1093/qmath/18.1.345.

[8] M. Mursaleen, "Matrix transformations between some new sequence spaces," Houston J. Math., vol. 9, no. 4, pp. 505-509, 1983.

[9] M. Mursaleen and O. Edely, "Statistical convergence of double sequences," J. Math. Anal. Appl., vol. 288, no. 1, pp. 223-231, 2003, doi: 10.1016/j.jmaa.2003.08.004.

[10] A. Pringsheim, "Zur theorie der zweifach unendlichen zahlenfolgen," Math. Ann., vol. 53, pp. 289-321, 1900, doi: 10.1007/BF01448977.

[11] G. Robison, "Divergent double sequences and series," Amer. Math. Soc. Trans., vol. 28, pp. 50-73, 1926, doi: 10.1090/S0002-9947-1926-1501332-5.

[12] E. Savaş, "On some new double lacunary sequence spaces via Orlicz function," J. Comput. Anal. Appl., vol. 11, no. 3, pp. 423-430, 2009.

[13] E. Savaş and R. Patterson, "Lacunary statistical convergence of multiple sequences," Appl. Math. Lett., vol. 19, pp. 527-534, 2006, doi: 10.1016/j.aml.2005.06.018.

[14] E. Savaş and R. Patterson, " $\left(A_{\sigma}\right)_{\Delta}$-double sequence spaces via Orlicz functions and double statistical convergence,” Iran. J. Sci. Technol., Trans. A, vol. 31, no. A4, pp. 357-367, 2007.

[15] B. Tripathy and H. Dutta, "On some lacunary difference sequence spaces defined by a sequence of Orlicz functions and $q$-lacunary $\delta_{m}^{n}$ statistical convergence," An. Şt. Univ. Ovidius Constanţa, vol. 20, no. 1, pp. 417-430, 2012.

Authors' addresses

Ayhan Esi

Adiyaman University, Science and Art Faculty, Department of Mathematics, 02040 Adiyaman, Turkey

E-mail address: aesi23@adiyaman.edu.tr

Hemen Dutta

Gauhati University, Mathematics Department, Guwahati, 781014 Assam, India

E-mail address: hemen_dutta08@rediffmail.com 